\title{
Impact of Gauge Representative Error on a Radar Rainfall Uncertainty Model
}

\author{
QIANG DAI \\ Key Laboratory of Virtual Geographic Environment, Ministry of Education, and School of Geography Science, \\ Nanjing Normal University, Nanjing, China, and Water and Environmental Management Research Centre, \\ Department of Civil Engineering, University of Bristol, Bristol, United Kingdom, and Jiangsu Center for \\ Collaborative Innovation in Geographical Information Resource Development and Application, Nanjing, China
}

\section{QIQI YANG}

Key Laboratory of Virtual Geographic Environment, Ministry of Education, and School of Geography Science, Nanjing Normal University, Nanjing, China

JUN ZHANG

Water and Environmental Management Research Centre, Department of Civil Engineering, University of Bristol, Bristol, United Kingdom

\section{SHULIANG ZHANG}

Key Laboratory of Virtual Geographic Environment, Ministry of Education, and School of Geography Science, Nanjing Normal University, Nanjing, China

(Manuscript received 30 September 2017, in final form 24 September 2018)

\begin{abstract}
In modeling the radar rainfall uncertainty, rain gauge measurement is generally regarded as the areal "true" rainfall. However, the inconsistent scales between radar and gauge may introduce a new uncertainty (also known as gauge representative uncertainty), which is erroneously identified as radar rainfall uncertainty and therefore called pseudouncertainty. It is crucial to comprehend what percentage of the estimated radar rainfall uncertainty actually stems from such pseudouncertainty rather than radar rainfall itself. For this reason, based on a fully formulated radar rainfall uncertainty model, this study aims to explore how the gauge representative error affects the distribution, spatial dependence, and temporal dependence of hourly accumulated radar rainfall uncertainty, and consequently affects the produced radar rainfall uncertainty band. Three group scenarios that delineate various degrees of gauge representative errors were designed to configure and run the uncertainty model. In the setting of a long-term analysis (almost 7 years) of the Brue catchment in the United Kingdom, we found that the gauge representative error affected the simulation of the marginal distribution of radar rainfall error, and had a considerable effect on temporal dependence estimation of radar rainfall uncertainty. The spread of the rainfall uncertainty band decreased with the growth of the gauge density in a radar pixel. The scenario with the lowest representative error only had $78 \%$ uncertainty spread of the scenario that has the largest error. This indicated there was a large impact of the representative error on radar rainfall uncertainty models. It is hoped that more catchments with diverse climate and geographical conditions and more radar data with various spatial scales could be explored by the research community to further investigate this crucial issue.
\end{abstract}

\section{Introduction}

Weather radars are subject to several sources of error, such as attenuation, ground clutter, occultation, and partial beam blocking. It is impractical to solve all of the error sources associated with radar rainfall measurement

Corresponding author: Shuliang Zhang, zhangshuliang@njnu.edu.cn step by step based on strict physical methods, not to mention complications introduced by the intricate interdependencies among these error sources (Ciach et al. 2007; Nikahd et al. 2016). Statistically modeling the uncertainty of radar rainfall measurement by simultaneously considering all its error sources is a promising alternative and a number of such methods have been proposed recently (Dai et al. 2014b; Germann et al. 2009; 
Kirstetter et al. 2010; Rico-Ramirez et al. 2015; Villarini et al. 2014). These methods are generally modeled by leveraging the strengths of rain gauge rainfall measurements, which is regarded as the "ground truth," toward the radar rainfall estimate (Dai and Han 2014; Krajewski et al. 2010). The discrepancy between radar and gauge rainfall measurements is interpreted as radar rainfall uncertainty and further partitioned into different error types, such as overall bias, conditional bias, and random error (Habib et al. 2008). The radar-gauge discrepancies at different locations and times may have different characteristics and diverse degrees of dependence may exist among them (AghaKouchak et al. 2010; Bringi et al. 2011; Villarini et al. 2014). Using different methods to describe and formulate these types of errors and the spatiotemporal dependence, a number of so-called radar rainfall uncertainty models have been proposed (Dai et al. 2014a,b; Germann et al. 2009; Habib and Qin 2013; Kirstetter et al. 2015; Rico-Ramirez et al. 2015; Seo et al. 2013).

In radar rainfall uncertainty models, it is a basic assumption that the discrepancies between the radar and gauge rainfall measurements all stem from the radar rainfall measurements. In other words, radar rainfall measurement uncertainty is assumed to be causing all of the discrepancy between the radar and gauge estimates. This premise obviously does not stand because it ignores the possible uncertainties produced by rain gauge measurements and inconsistent conditions in comparing radar and gauge measurements. Gauge rainfall measurements are also subjected to many error sources, such as windinduced error, evaporation loss, and raindrop splashing (Ciach 2003; Habib et al. 1999; Michelson 2004; Villarini et al. 2008), but this uncertainty can be confined to an acceptable level after careful quality control and can be ignored when estimating radar rainfall uncertainty.

The other type of uncertainty is derived from inconsistent conditions when comparing radar and gauge measurements, which is disregarded in most uncertainty models. For example, because radar observes rainfall aloft while a rain gauge measures the rainfall on the ground, radar-gauge rainfall discrepancies can be caused by various microphysics processes (e.g., evaporation, drift, aggregation, melting, breakup, collision, and coalescence) when hydrometeors descend from the radar observation height to the ground (Dai and Han 2014; Wang et al. 2015). A number of studies have proposed ways of tackling these issues (Dai et al. 2013; Qi et al. 2013; Sandford 2015; Hall et al. 2015; Xie et al. 2016).

The so-called inconsistent condition also refers to the radar-gauge spatial-scale inconsistency. Rain gauge data are generally reported as point rainfall or on the scale of decimeters, while weather radar is sampled on the scale of kilometers or hundreds of meters (e.g., $2 \mathrm{~km}$ in this study). The error induced by inconsistent radargauge scales is also well known as gauge representative error (Bringi et al. 2011; Hasan et al. 2014), which has been explored in previous studies (Ciach and Krajewski 1999a; Habib et al. 2004). For example, Barnston (1991) proposed a so-called error variance separation (EVS) method, which was adopted and improved by Ciach and Krajewski (1999a) and Ciach (2003). The key concept of the EVS is to partition the radar-gauge difference variance into the radar rainfall estimation error variance and the representative error variance. There are numerous successive studies striving to apply or improve this scheme (Anagnostou et al. 1999; Bringi et al. 2011; Kirstetter et al. 2010). There is special concern on the gauge representative error in merging radar and gauge rainfall measurements (Hasan et al. 2016a,b). It has been shown that $50 \%-80 \%$ of the discrepancy between radar rainfall estimates and rain gauge rainfall can be induced by rain gauge representative error for hourly rainfall totals with a grid size of $3 \mathrm{~km}$ (Ciach and Krajewski 1999a).

Despite its vital importance, the inconsistent scales issue is not considered or addressed in most up-to-date radar rainfall uncertainty models (Germann et al. 2009; Kirstetter et al. 2015; Rico-Ramirez et al. 2015; Villarini et al. 2014). Compared with the traditional uncertainty model with a simple structure, recent models have complicated frameworks and consist of many components. For example, in traditional models, only the differences or ratios between the radar and gauge rainfall are estimated to represent radar rainfall uncertainty (Anagnostou et al. 1999; Borga et al. 2002; Ciach and Krajewski 1999b; Habib et al. 2008). In recent models, some significant factors such as the distribution and the spatial and temporal dependence of rainfall errors are recognized, and complicated methods have been established to investigate them. It is a larger challenge if the radar-gauge scale inconsistencies are further considered in such advanced models.

Despite this, we believe that even if the representative error in a radar rainfall uncertainty model cannot be solved completely, it is necessary to understand whether and how this error changes the outcomes of the uncertainty model. In other words, one should know how much of the estimated radar rainfall uncertainty actually stems from the gauge representative error, rather than from radar rainfall itself, which we refer to as radar rainfall pseudouncertainty. For this reason, based on the previous proposed radar rainfall uncertainty model, this study aims to explore the relationship between gauge representative error and the distribution, spatial dependence, and temporal dependence of radar rainfall uncertainty. Moreover, this study will investigate the percentage of the estimated radar rainfall uncertainty 
Brue Catchment

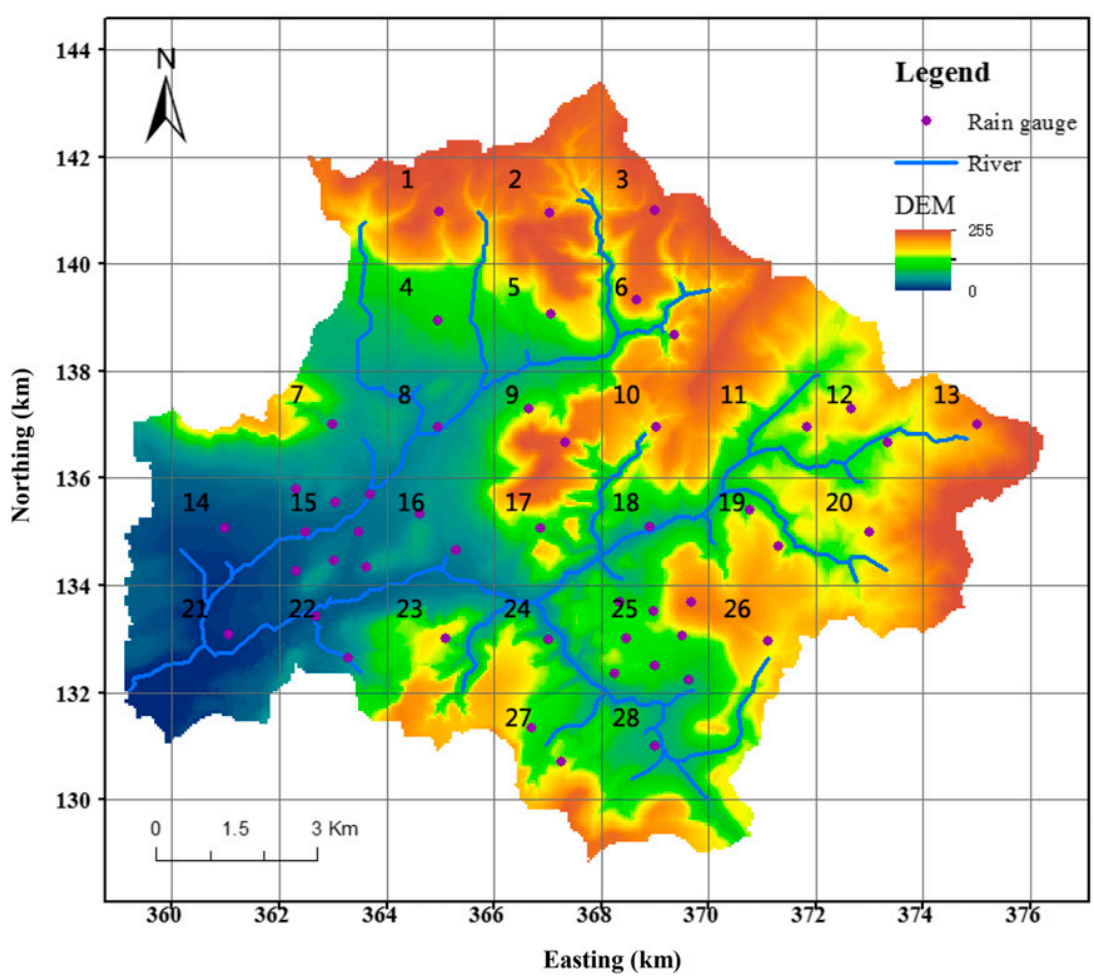

Pixel 15

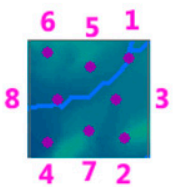

Pixel 25
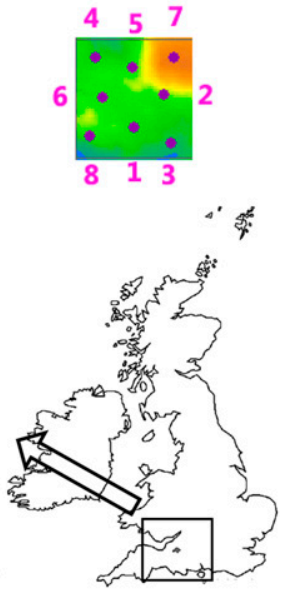

FIG. 1. Map of the rain gauges (purple dots) and the Brue catchment based on the British National Grid coordinate system. The digital elevation model (DEM; $\mathrm{m}$ ) is shown in the background. The grids represent the radar pixels, whose indices are also shown on the grid. The gauge indices of two radar pixels (pixels 15 and 25) are also specified.

that is due to gauge representative error. This study is the first to investigate the impact of gauge representative error on radar rainfall uncertainty models and can contribute to the understanding of uncertainty associated with radar rainfall.

This paper is organized as follows. After the introduction, section 2 illustrates the study area, data sources, and algorithm of the uncertainty model. The experimental design scheme is also introduced. Section 3 presents the results of uncertainty models established under different scenarios, and section 4 shows the impact of gauge representative error on the radar rainfall uncertainty band. A detailed discussion of these results is given in section 5 . Finally, the section 6 summarizes the key findings and limitations and outlines future work.

\section{Data sources and methods}

\section{a. Study area and data sources}

Rain gauge network density and its distribution are important factors that determine the analysis quality of this study. The Brue catchment located in Somerset in southwest England is used herein. Within an area of
$135 \mathrm{~km}^{2}, 49$ tipping-bucket rain gauges (TBRs) with $0.2-\mathrm{mm}$ resolution are reasonably distributed. Here, reasonably mainly refers to the distribution of rain gauges taking careful account of the radar pixels (see Fig. 1 and section 2c). The radar datasets used were collected from the Hydrology Radar Experiment (HYREX). The C-band radar is located at National Grid Reference 36091023 at an elevation of $255 \mathrm{~m}$ with a range of approximately $40 \mathrm{~km}$ from the center of the catchment. The basic parameter settings for the weather radar used in this study are listed in Table 1. The radar completes one cycle through four different scan elevations $\left(0.5^{\circ}, 1.0^{\circ}, 1.5^{\circ}\right.$, and $2.5^{\circ}$ ) every $5 \mathrm{~min}$. The elevation of the radar beam for the lowest angle $\left(0.5^{\circ}\right)$ is approximately $400 \mathrm{~m}$ above ground level. The radar data were processed to correct the attenuation. The gridded radar rainfall values were estimated from the polar radar reflectivity values using a suite algorithm (Collier and James 1986). The terrain elevation of the Brue catchment is less than $255 \mathrm{~m}$, so there should not be any terrain blockage. Twenty-eight radar pixels with $2-\mathrm{km}$ resolution cover most of the catchment area. The Marshall-Palmer $Z-R$ relationship (Marshall et al. 1955) is applied to convert radar reflectivity into rainfall intensity: 
TABLE 1. Basic parameter settings for the weather radar used in this study.

\begin{tabular}{ll}
\hline \hline Radar parameters & \multicolumn{1}{c}{ Settings } \\
\hline Location & $50.82^{\circ} \mathrm{N}, 2.56^{\circ} \mathrm{W}$ (Wardon Hill, England) \\
Elevation & $255 \mathrm{~m}$ \\
Wavelength & $\mathrm{C}$ band $(5 \mathrm{~cm})$ \\
Range & $210 \mathrm{~km}$ \\
Beamwidth & $1^{\circ}$ \\
Scan elevations & $0.5^{\circ}, 1.0^{\circ}, 1.5^{\circ}, 2.5^{\circ}$ \\
Scan time & $5 \mathrm{~min}$ \\
Scan type & PPI \\
\hline
\end{tabular}

$$
Z=200 R^{1.6}
$$

where $Z$ and $R$ refer to radar reflectivity and rainfall intensify, respectively. The relationship is the standard equation used in the United Kingdom (Bringi et al. 2011; Dai et al. 2017; Rico-Ramirez et al. 2007). Descriptions of the radar data processing can be found in previous studies (Wheater et al. 2000; Wood et al. 2000a,b). The 5-min rainfall estimates were accumulated to create an hourly dataset. The hourly radar and gauge datasets cover the period from 1993 to 2000. Among them, the datasets from 1993 to 1998 were used to estimate the parameters of the uncertainty model, while the remaining data were used for validation. In particular, a month-long period from August 1999, when there were large storms, was specially chosen for evaluation.

Despite the fact that the radar and rain gauges document historical rainfall, the Brue catchment is still an ideal area for this study. The rain gauge network was designed by considering the locations of radar pixels, thus ensuring the density and distribution of rain gauges are quite suitable for analyzing the radar-gauge inconsistent scale issue, and also the radar and gauge datasets are trustworthy because of careful quality control (Bringi et al. 2011; Moore et al. 2000). The rain gauge network, radar pixels, boundary of the Brue catchment, and the terrain are shown in Fig. 1. The 28 radar pixels within the catchment are mainly used in this work, and their indices are labeled in the figure.

\section{b. Radar rainfall uncertainty model}

The empirically based uncertainty model is a practical way of describing complicated and interdependent radar rainfall uncertainties. It aims to explore the most significant features of radar rainfall from the long-term historical data and extend them into the future state. For example, the overall bias of radar rainfall compared with reference rainfall is an effective uncertainty analysis method and can serve as a fundamental conceptual framework for other complicated uncertainty models.
Furthermore, there are some uncertainty models that aim to describe diverse features of rainfall uncertainty simultaneously (Dai et al. 2014a; Villarini et al. 2014). To better illustrate the uncertainty of radar rainfall and its propagation through a hydrological model, an ensemble generator for radar rainfall can be established (Ciach et al. 2007; Germann et al. 2009). In this study, we adopt the previously presented uncertainty model and ensemble rainfall generator from Dai et al. (2014b) to examine the possible impact of inconsistent spatial scales on radar rainfall uncertainty. The radar rainfall uncertainty illustrates the additive difference of radar rainfall toward the reference rainfall for different rainfall intensities. The introduced ensemble generator, called the Multivariate Distributed Ensemble Generator (MDEG), is driven by the copula and autoregressive filter, which is used to produce a certain number of probable "true" rainfall fields by adding a corresponding number of random fields to the deterministic component of the rainfall. A basic assumption of this generator is that the gauge rainfall can provide a sufficiently accurate approximation of the true rainfall for a radar pixel. The possible discussion under this assumption has been studied previously in the literature (Bringi et al. 2011; Ciach and Krajewski 1999a; Habib et al. 2004).

There are three crucial characteristics of radar rainfall uncertainty investigated by the uncertainty model, namely probabilistic distribution, spatial dependence, and temporal dependence of radar rainfall uncertainty. The model works proceeds along the following steps. Primarily, the probability distribution of true rainfall, once given a radar estimate, was simulated, which revealed the degree of confidence in the radar products. As the rainfall uncertainty changes with the rain rates (Dai et al. 2014b), the relationship between the probability distribution and rainfall intensity was also investigated. To better integrate the distribution with other components of the uncertainty model, the observed distribution was fitted with five traditional distribution models: Gaussian, lognormal, gamma, Weibull, and generalized extreme value (GEV). The parameter structures of these models are given in Table 2. Except for the GEV model, these distributions have two parameters (see Table 2). The additional parameter in GEV increases the complexity of the uncertainty model. In addition, as the Gaussian distribution is more tractable via statistics, it can better handle the incorporation of the rainfall uncertainty model and the hydrometeorological system. However, there is an inevitable problem with the Gaussian model, as it ignores the fact that the rainfall must be positive. Whether the inconsistent scale effect affects the distribution shape of the rainfall uncertainty will be explored in section 3. In the second step, the spatial interdependence of rainfall uncertainty among radar pixels 
TABLE 2. Parameters of distributions used in this study.

\begin{tabular}{lccc}
\hline Distribution & Location & Scale & Shape \\
\hline Gaussian & $\times$ & $\times$ & \\
Lognormal & $\times$ & $\times$ & \\
Gamma & & $\times$ & $\times$ \\
Weibull & & $\times$ & $\times$ \\
GEV & $\times$ & $\times$ & $\times$ \\
\hline
\end{tabular}

was simulated based on the copula method. To avoid the possible influence of copula functions, both Gaussian and $\mathrm{t}$ copulas were used. Finally, the presented uncertainty model was used to calculate the temporal dependence of rainfall uncertainty for different lag times. The lag time was set from 1 to $5 \mathrm{~h}$.

Based on the above uncertainty model, we can generate a given number of rainfall ensemble members, which is of great importance if we want to integrate the radar rainfall with other models and systems such as the hydrological model, data assimilation system, and rainfall nowcasting system. The ensemble generator can fully formulate the various components of the uncertainty model, while at the same time offering simple outcomes that can be easily utilized. An ensemble generator named MDEG was employed in this study (Dai et al. 2014b). MDEG was established based on conditional distribution, copula, and autoregressive (AR) models, and it can generate a massive number of probable true surface rainfall fields with their natural spatiotemporal structure to represent the radar rainfall uncertainty. A detailed description of MDEG is given by Dai et al. (2014b).

\section{c. Experimental design}

The development of a framework that helps us understand the impacts of representative error on a radar rainfall uncertainty model requires the design of several experiments that collectively provide meaningful insights into how the components of a radar rainfall uncertainty model are relevant to the problems of a radar-gauge inconsistent scale.

As mentioned above, the major scale concern in this study originates from the fact that a rain gauge measures rainfall at the scale of a decimeter, while radar measures at the scale of kilometers or hundreds of meters. However, this does not mean we need a number of gauges that can be evenly distributed and cover the entire study area. This is because rainfall is not randomly distributed in space and has close spatial autocorrelation; consequently, the intermittent distribution of rain gauges can also reflect the rainfall variation over the whole area. Except in specially designed experimental areas, rain gauge placement is quite sparse in real catchments. For most catchments around the world, it is impossible to ensure there is at least one rain gauge for each radar pixel. Theoretically speaking, an increase in the number of rain gauges within a radar pixel could reduce the gauge representative error when considering the sparse gauge density in most areas. However, this statement assumes that the impact of gauge distribution and topography can be disregarded. In the Brue catchment, because the rain gauges are specifically designed for weather radar research, the distribution of gauges is quite even within each pixel (see pixels 15 and 25 in Fig. 1). Moreover, the terrain is relatively gentle within each radar pixel in most areas of the Brue catchment. For this reason, there is a certain corresponding relationship between gauge density and gauge representative error for each radar pixel in the Brue catchment.

Based on the experimental configuration of the rain gauges, the radar pixels in the Brue catchment were partitioned into three groups according to their gauge densities. As can be seen in Fig. 1,28 radar pixels (almost covering the catchment) each have one rain gauge. The gauge number increases to two along two parallel southwest-northeast lines across the catchment, aligned with the prevailing wind direction and running from lowland to upland (Bell and Moore 2000). More attractively, there are two superdense radar pixels with eight gauges (pixels 15 and 25). Based on this, three groups of scenarios describing different degrees of gauge representative uncertainty were developed (see Table 3). Group A contains eight scenarios that correspond to 28 radar pixels, each with one rain gauge, which is the standard way of establishing the radar rainfall uncertainty model. With only one gauge in each radar pixel, the derived rainfall uncertainty using by this scheme is likely to experience large representative error. As eight gauges are contained in pixels 15 and 25, we sequentially selected one gauge from each pixel to form a scenario together with the other 26 radar pixels (see Table 3 ). Comparison of the eight scenarios is an effective way of exploring the impact of gauge location within a radar pixel on characteristics of estimated radar rainfall uncertainty.

Group B, with two scenarios, focuses on nine radar pixels that have at least two rain gauges (pixels $6,9,12$, $15,16,19,22,25$, and 27). Specifically, scenario B1 only used one gauge in each pixel to build a radar rainfall uncertainty model while scenario B2 used two of them. The gauge number in each radar pixel is specially defined herein as gauge density, so scenarios B1 and B2 are also called one-gauge-density and two-gauge-density scenarios, respectively. Comparison of scenarios B1 and B2 will reveal the changes of estimated rainfall uncertainty when there are two gauges in a radar pixel.

Group C, which consists of eight scenarios, was designed to further investigate the relationship between gauge 
TABLE 3. Configuration scenarios of rain gauge and radar pixel network with different gauge densities (number of gauges within a radar pixel).

\begin{tabular}{cccccc}
\hline \hline Group & Scenario & Gauge density & Total pixel count & Gauge index in pixel 15 & Gauge index in pixel 25 \\
\hline A & A1 & 1 & 28 & 1 & 1 \\
& A2 & 1 & 28 & 2 & 2 \\
& A3 & 1 & 28 & 3 & 3 \\
& A4 & 1 & 28 & 4 & 5 \\
& A5 & 1 & 28 & 5 & 6 \\
& A6 & 1 & 28 & 7 & 7 \\
& A7 & 1 & 28 & 8 & 1 \\
& A8 & 1 & 9 & 1 & $1-2$ \\
B & B1 & 1 & 9 & $1-2$ & 1 \\
& B2 & 2 & 2 & 1 & $1-2$ \\
C & C1 & 1 & 2 & $1-2$ & $1-3$ \\
& C2 & 2 & 2 & $1-3$ & $1-5$ \\
& C3 & 3 & 2 & $1-4$ & $1-6$ \\
& C4 & 4 & 2 & $1-5$ & $1-7$ \\
& C5 & 5 & 2 & $1-7$ & $1-8$ \\
\hline
\end{tabular}

representative error and radar rainfall uncertainty. All of these scenarios concentrate on pixels 15 and 25, and vary the gauge number in a pixel from one to eight (corresponding to scenarios $\mathrm{C} 1-\mathrm{C} 8$ ). The gauge density and total number of pixels for all scenarios are listed in Table 3. If the original radar pixel has more gauges than the required number of the given scenario, a random selection process was carried out to remove the excess number of gauges.

For all designed scenarios, the long-term radar and gauge pairs were first established. The average rainfall measured by the rain gauges was calculated if there was more than one gauge in a radar pixel. The possible loss of the average form for analyzing the representative error will be demonstrated in the discussion section. MDEG was then configured to calculate the probabilistic distribution, spatial dependence, temporal dependence, and probabilistic bands of radar rainfall uncertainty based on corresponding radar and gauge datasets. There are also special configurations when dealing with different types of scenarios, which will be discussed in the following section.

\section{Radar rainfall uncertainty modeling under different scenarios}

\section{a. Group A scenario}

The uncertainty model used herein has to simulate three components of hourly accumulated radar rainfall uncertainties, namely marginal distribution, spatial dependence, and temporal dependence. The datasets from 1993 to 1998 were used to estimate the parameters of the uncertainty model, while the remaining datasets covering 1999 and 2000 were used for validation.

Scattergraphs of the radar-gauge comparisons at the hourly time scale for nine radar pixels are shown in Fig. 2. The radar pixels (e.g., pixels 1, 2, 3, 13, 14, 20, 21, 26 , and 28) located at the boundary and center catchment are chosen. The numbers of radar-gauge pairs are plotted with different colors. An obvious discrepancy between radar and gauge rainfall is observed, which is acceptable as a long-term dataset with a variety of rainfall situations included. An overestimation by the radar when compared to gauges is observed for all radar pixels.

The marginal distribution refers to the probabilistic conditional distribution of gauge rainfall once given a radar rainfall estimate. A GEV model was adopted to model the marginal conditional distribution for each radar pixel. The detailed comparison of fitting effects by different theoretical models is given in the following section. The GEV model has three parameters, namely, location $\mu$, scale $\sigma$, and shape $k$. Because the conditional distribution has a close relationship with rain rate $r$, the location and scale parameters are parameterized using power-law functions (Dai et al. 2014b):

$$
\begin{aligned}
& \mu(p)=a_{\mu} r(p)^{b_{\mu}} \quad \text { and } \\
& \sigma(p)=a_{\sigma} r(p)^{b_{\sigma}}
\end{aligned}
$$

where $p$ refers to a radar pixel and $a$ and $b$ are coefficients of fitted functions with the subscript representing the 

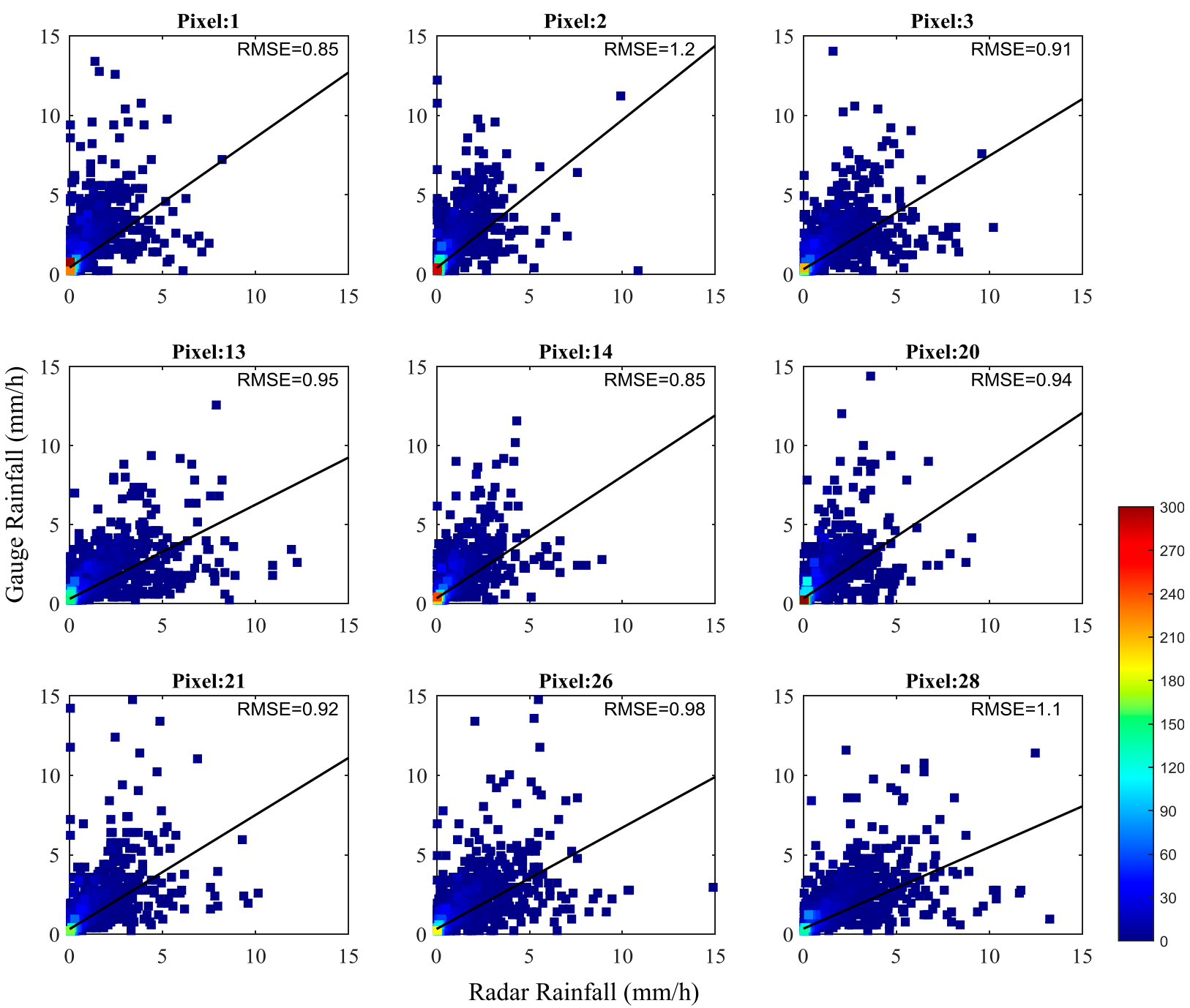

FIG. 2. Scattergraph of the radar-gauge comparisons at the hourly time scale for nine radar pixels. The numbers of radar-gauge pairs are plotted with different colors.

GEV parameters. Because the marginal distribution is conditioned on the rain rate $r$, the difference between $r$ and the GEV location parameter $\mu$ can to some extent reveal the systematic bias of the radar rainfall. In addition, the scale $\sigma$ refers to the spread of rainfall errors, which we call the deviation of the random error. The estimated parameters for radar pixels 15 and 25 are plotted in Fig. 3. Figure 3 shows the location and scale parameters with rain rates for the eight scenarios. For example, take the location parameters in radar pixel 15; eight rain gauges located in this pixel are separately used to estimate the marginal distribution of radar rainfall uncertainty. The largest, smallest, and mean values of the location parameters for each rain rate are depicted. A remarkable variation of location parameters is observed, which means even within a small domain of
$2 \times 2 \mathrm{~km}^{2}$, a slight shift in gauge location will produce a clear change among the location parameters. The same conclusion can be drawn for the other two GEV parameters (see Fig. 3).

The spatial interdependence among radar pixels is shown in Fig. 4. The correlation coefficients are estimated using Gaussian and t copula functions. Because the spatial correlation coefficients are quite consistent among the different scenarios in group A, only scenario A1 is displayed. In Fig. 4, each gray dot delineates the relationship between the spatial correlation coefficient between the two radar pixels and their separated distance. To better demonstrate this relationship, the scattered points are fitted with a three-parameter exponential function (black curve in Fig. 4. Another core component of the uncertainty model, temporal 
Pixel 15
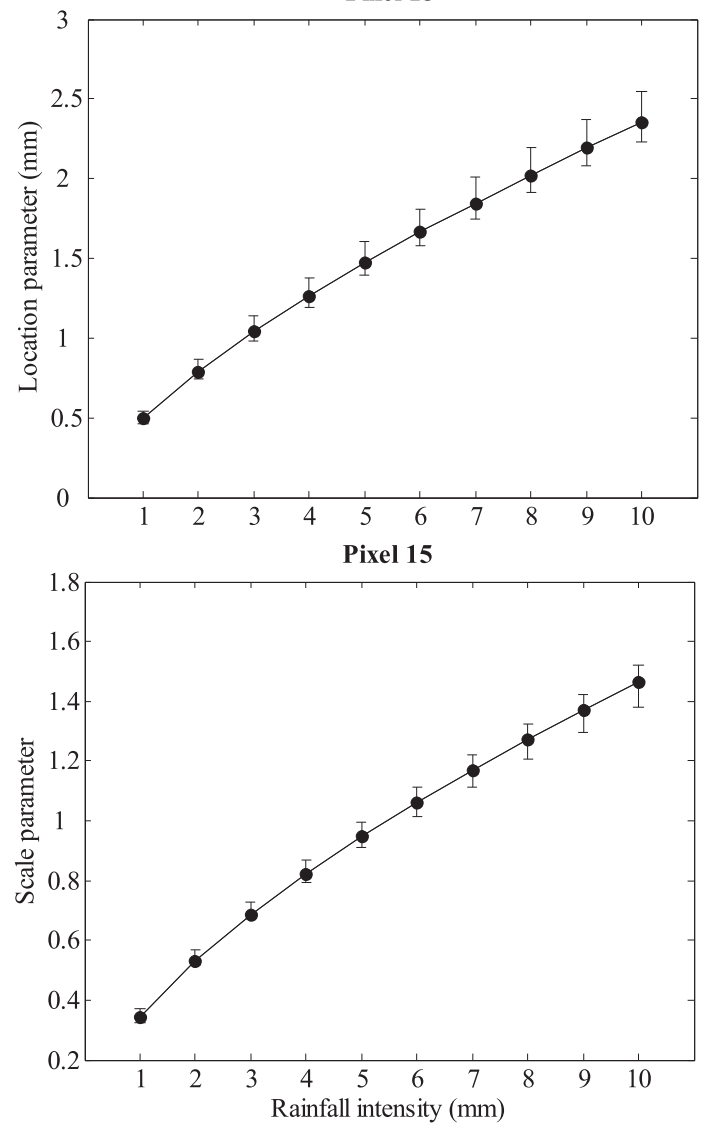

Pixel 25
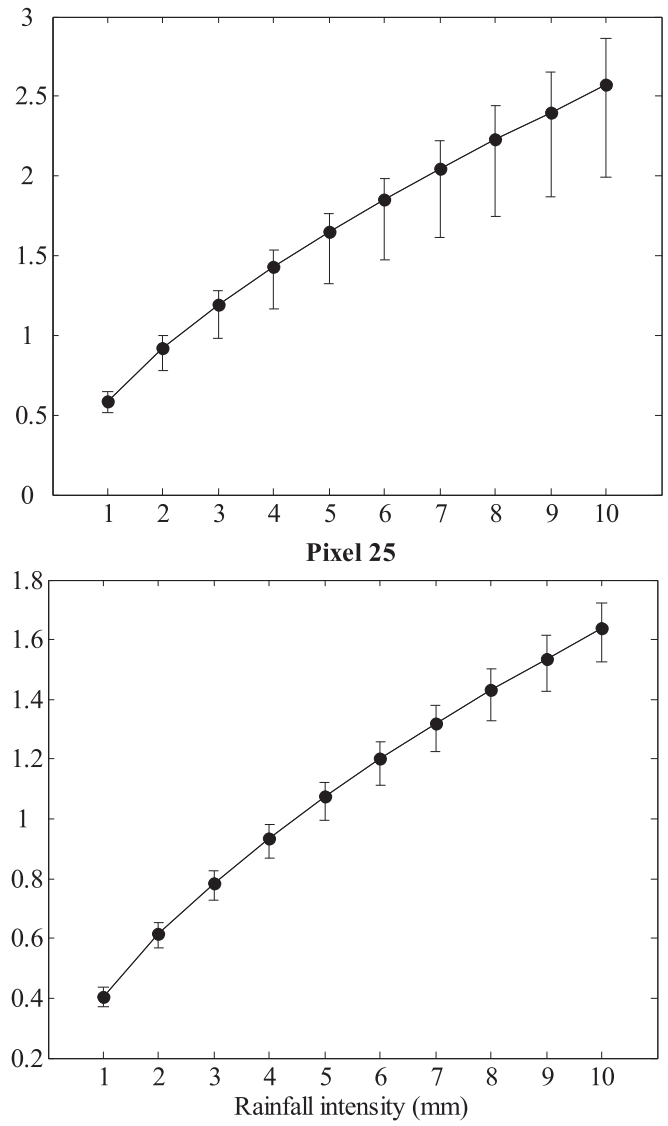

FIG. 3. Variability of the location and scale parameters with respect to hourly rainfall intensity for group A scenarios.

dependence, is simulated for scenarios A1-A8 and depicted in Fig. 5. As for the spatial dependence, both the Gaussian and $t$ copula functions are used. As shown in Fig. 5, the temporal correlation of rainfall uncertainty is generally weak for all lag hours and decreases considerably from lag hours 1 to 5 . The variations of the temporal correlation coefficients among the different scenarios are considerable.
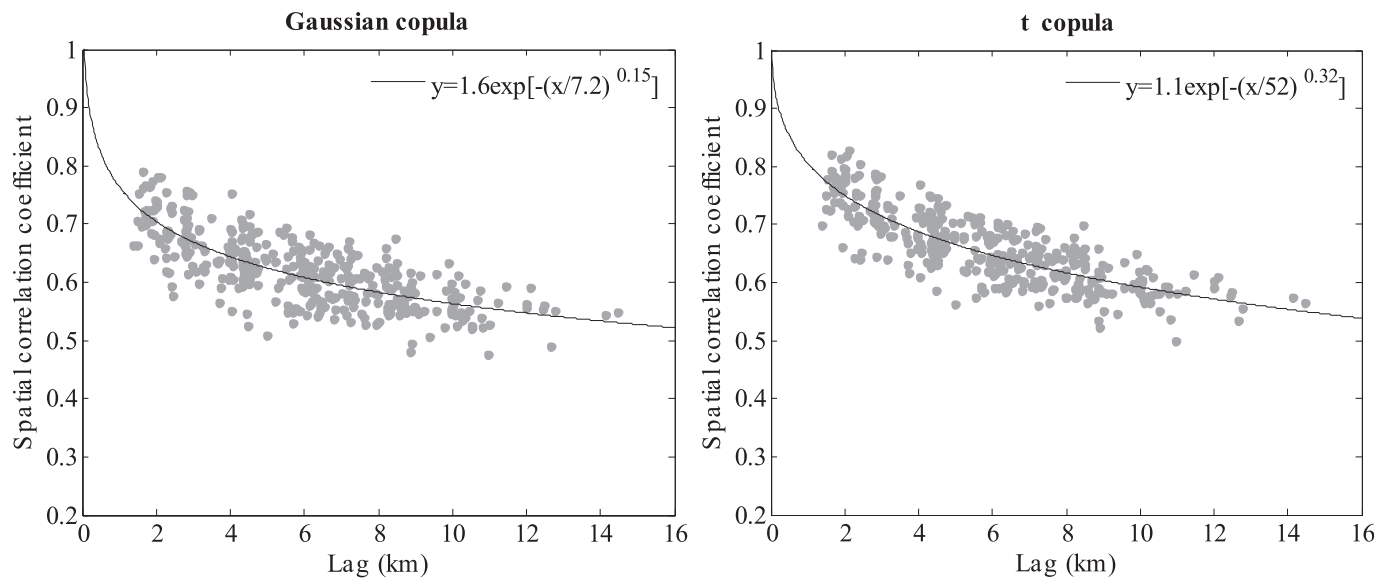

FIG. 4. The relationship between spatial correlation coefficients of the radar rainfall errors and lag distances using (left) Gaussian and (right) t copulas. 

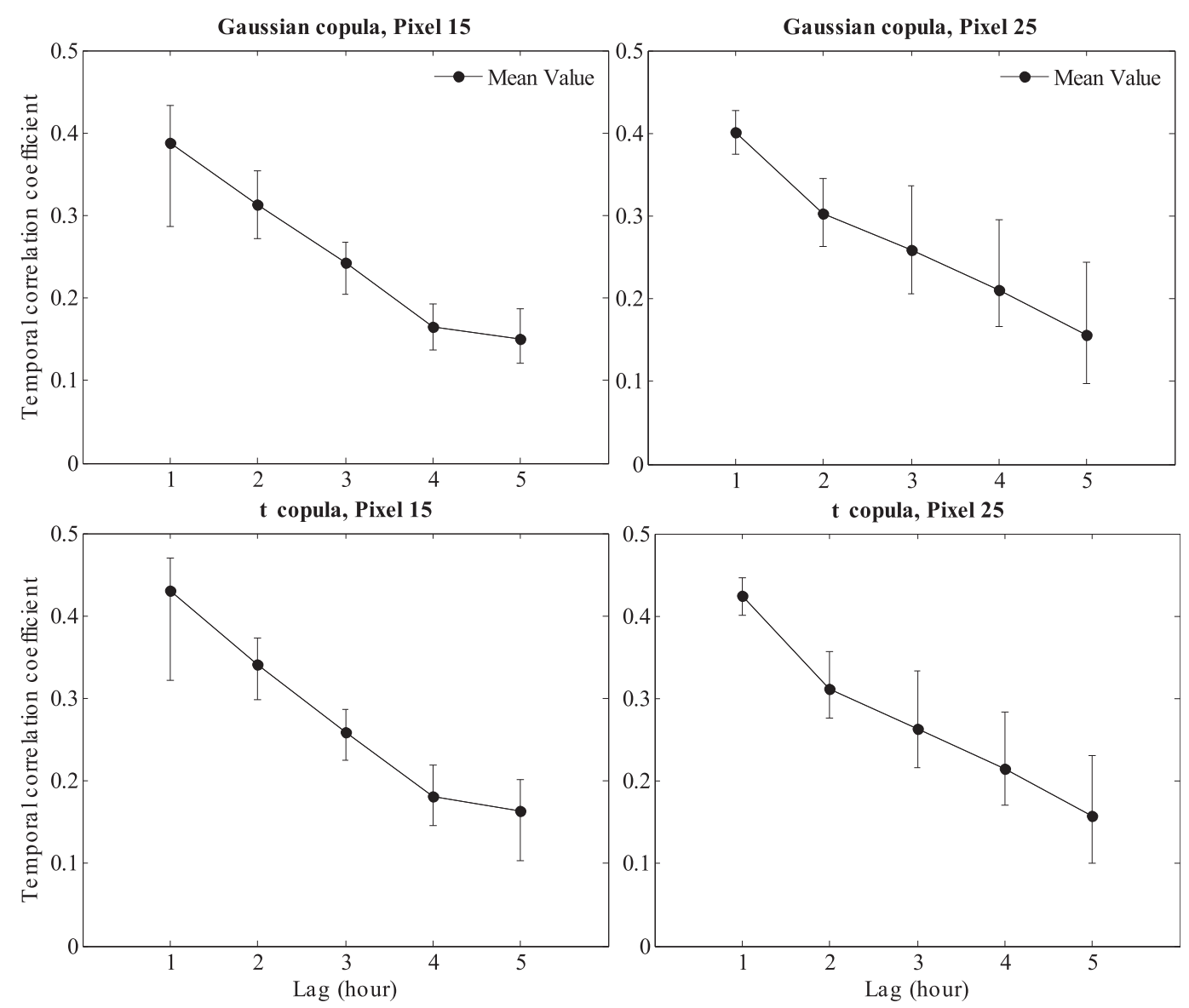

FIG. 5. Variability of temporal correlation coefficients with lag hours for group A scenarios.

\section{b. Group B scenarios}

Group B scenarios focus on nine radar pixels that have two rain gauges. As mentioned above, the conditional marginal distribution could be modeled with diverse theoretical distribution models. The chosen theoretical model is of great importance as it determines the systematic and random error of the radar rainfall. For this reason, a carefully fitted test should be used to choose the best distribution for modeling the radar rainfall uncertainty. More importantly, it is important to know whether the gauge representative error will affect this appraisal.

The chi-squared test is a general test used to compare the observed cumulative distribution function (CDF) to the expected one. To avoid any possible influence of model complexity (e.g., a three-parameter model tends to fit better than a two-parameter model) on the evaluated outcomes, a tenfold cross-validation scheme was adopted. Specifically, nine-tenths of the calibrated datasets were used to fit the observed data with a distribution model, and one-tenth was left for computing the chi-squared indicator using the fitted parameters. This process was repeated 10 times and the mean results (named the fitness index) were finally used to evaluate the fitness between the observed data and the given distribution. The higher index corresponds to the inferior performance of the distribution models. The fitness indices for pixels 15 and 25 are shown in Tables 4 and 5 (these two radar pixels were chosen because they are used in all of the scenarios). Because the rain rate will affect the distribution of the radar uncertainty, the above test process was carried out for various rain rates, namely, $0-3,3-5$, and $>5 \mathrm{~mm}$. This classification can represent different magnitudes of rain-rate conditions and ensure each subsample has enough data for the subsequent analysis. As shown in Table 4, the fitting indices increase with the increase in the rain rate, and the Gaussian distribution shows the worst performance for all cases, while the GEV and the lognormal distributions are the best. Specifically, the GEV model is the best for cases of 0-3 and 3-5 mm, while the lognormal approach yields the same performance as the GEV when 
TABLE 4. Fitting indices using different distributions for radar pixel 15.

\begin{tabular}{clcccrr}
\hline \hline Rainfall $(\mathrm{mm})$ & Scenario & Gaussian & Lognormal & Gamma & Weibull & GEV \\
\hline \multirow{2}{*}{ 0-3 } & B1 & 0.10 & 0.05 & 0.07 & 0.07 & 0.05 \\
& B2 & 0.12 & 0.05 & 0.07 & 0.07 \\
$3-5$ & B1 & 0.12 & 0.10 & 0.10 & 0.10 & 0.09 \\
& B2 & 0.15 & 0.10 & 0.11 & 0.11 & 0.09 \\
& B1 5 & B2 & 0.20 & 0.16 & 0.15 & 0.17 \\
\end{tabular}

the rain rate is greater than $5 \mathrm{~mm}$. When comparing scenario $\mathrm{B} 2$ to scenario $\mathrm{B} 1$, there are considerable changes in the fitness indices for the different cases. Taking the GEV model for example, the fitting index is 0.17 in scenario B1 when the rain rate is greater than $5 \mathrm{~mm}$, while it is only 0.14 for scenario B2. However, both scenarios indicate that the GEV model is the optimum one in fitting the observed distribution. From this viewpoint, the GEV model was selected for the following calculations and analysis. Table 5 for pixel 25 further supports this decision. More importantly, we found that the increase in the rain gauge density (from one-gauge density to two-gauge density) does not significantly affect the chosen or marginal distribution model for radar rainfall uncertainty.

If for safety we leave out the distribution type, the estimated parameters of the distribution may have a close relationship with the gauge representative error. For this reason, the fitting parameters of the GEV model (all distribution parameters refer to the GEV model parameters in the following analysis unless otherwise specified) are further compared between the two scenarios. The location and scale parameters are drawn against the rain rate for scenarios $\mathrm{B} 1$ and $\mathrm{B} 2$, as shown in Fig. 6. Two locations (pixels 15 and 25) are delineated for comparison. In general, the positive location parameter indicates the radar rainfall measurements are overestimated against the rain gauge rainfalls. It is unsurprising to see that the location parameter grows with the increase in rain rate. The differences in the location parameters between scenarios B1 and B2 are quite small.

In terms of the scale parameters of the GEV model, it is clear that the parameters of scenario B1 are larger than those of $\mathrm{B} 2$ over the entire range of rain rates. The largest difference between them was approximately 0.25 (when rainfall was equal to $10 \mathrm{~mm}$ for pixel 15), which accounted for about $20 \%$ of scenario B2. However, with the same radar rainfall measurements, the uncertainties associated with radar rainfall modeling in scenario B1 seem to be larger than those in scenario B2. In terms of the shape parameter (see Table 6), the ones in scenario B2 are generally smaller than those in scenario B1 for all nine radar pixels. Because the GEV shape parameter to some extent reveals the skewness of the distribution shape, it indicates that the marginal distribution under scenario B1 tends to be more asymmetric.

A comparison of the interdependence among nine radar pixels between scenarios B1 and B2 is shown in Fig. 7. As was done for the group A scenarios, the spatial dependence was estimated by the Gaussian and $t$ copula methods. It is observed that the correlation coefficients are all within $0.5-0.8$, which are almost the same as those of scenario A. Specifically, the spatial coefficients of scenario B2 are a bit smaller than those of scenario B1, but their overall trends are almost the same, which can be proved by the regressive three-parameter exponential function. The temporal coefficients of two scenarios for radar pixels 15 and 25 using a Gaussian copula approach are shown in Fig. 8. One can observe from Fig. 8 that scenario B2 has a higher temporal correlation than scenario B1 for all lag hours. From the location of radar pixel 15, the differences are more significant when the separated time is confined to $2 \mathrm{~h}$. In summary, a two-gauge-density scenario does induce some changes in modeling the spatial and temporal dependence of radar rainfall uncertainty

TABLE 5. Fitting indices using different distributions for pixel 25.

\begin{tabular}{|c|c|c|c|c|c|c|}
\hline Rainfall (mm) & Scenario & Gaussian & Lognormal & Gamma & Weibull & GEV \\
\hline \multirow[t]{2}{*}{$0-3$} & B1 & 0.09 & 0.05 & 0.07 & 0.06 & 0.05 \\
\hline & B2 & 0.10 & 0.04 & 0.06 & 0.06 & 0.03 \\
\hline \multirow[t]{2}{*}{$3-5$} & B1 & 0.12 & 0.10 & 0.10 & 0.10 & 0.10 \\
\hline & $\mathrm{B} 2$ & 0.13 & 0.10 & 0.10 & 0.10 & 0.09 \\
\hline \multirow[t]{2}{*}{$>5$} & $\mathrm{~B} 1$ & 0.20 & 0.19 & 0.19 & 0.19 & 0.19 \\
\hline & $\mathrm{B} 2$ & 0.22 & 0.20 & 0.20 & 0.20 & 0.19 \\
\hline
\end{tabular}


Pixel 15
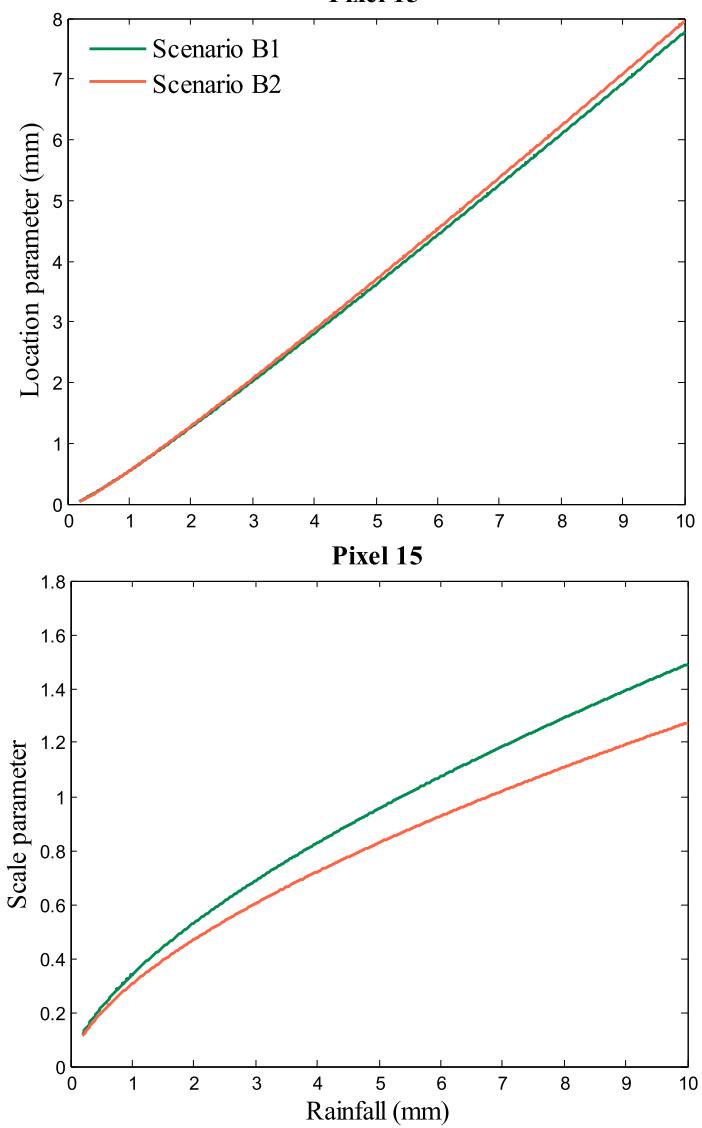

Pixel 25
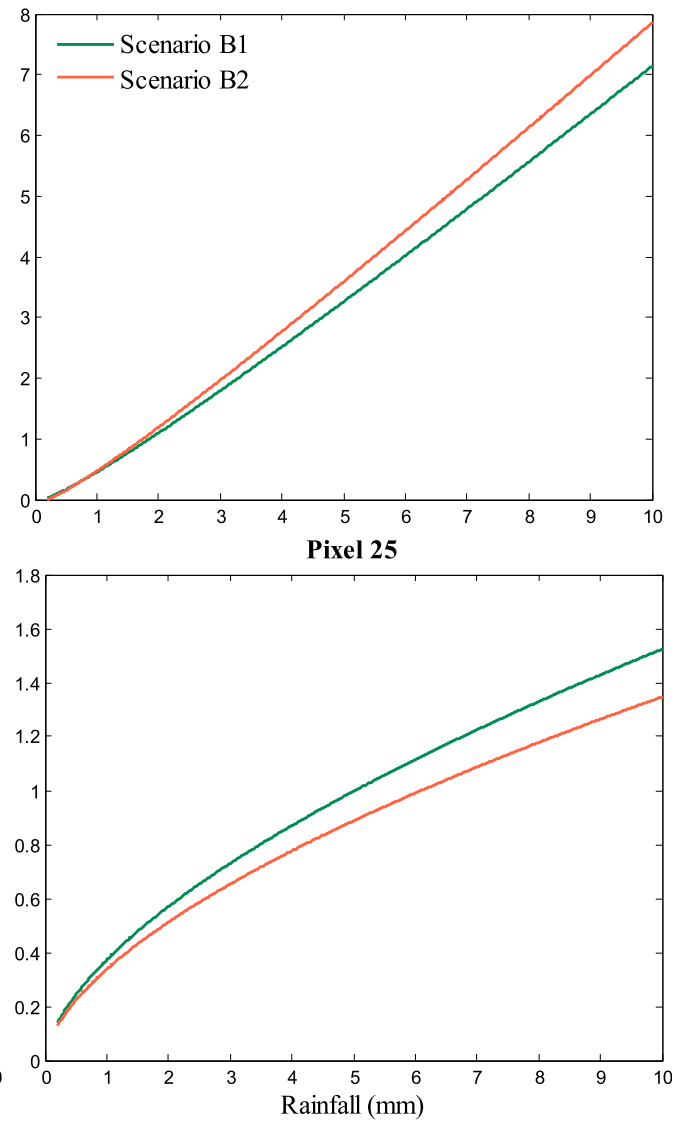

FIG. 6. Location and scale parameters of hourly radar rainfall estimates in two radar pixels for group B scenarios.

compared to the one-gauge-density scenario, especially for temporal dependence.

\section{c. Group C scenarios}

To obtain a reliable relationship between gauge density per pixel and representative-induced pseudouncertainty, the gauge density was set from one gauge to eight gauges in the group $\mathrm{C}$ scenarios. The overall correlation between long-term hourly radar and gauge rainfall measurements were calculated first (see Table 7). It is worth noting that only rainy hours were selected. Because radar and gauge rainfall values have a nonlinear relationship (their discrepancy increases with the growth of the rain rate), Kendall's rank correlation coefficients were used here. It can be seen in Table 7 that there is an obvious enhancement of the correlation between radar and gauge rainfall amounts with an increase in gauge density, indicating the discrepancy between the radar and gauge datasets is not all induced by radar rainfall measurements.

The systematic error and deviation of random error for group $\mathrm{C}$ scenarios were estimated. Because there was almost no change in the systematic error among the different scenarios, only the deviation of random error is shown in Fig. 9. A declining trend in the deviation of random errors with an increase in gauge density is visible in Fig. 9. This observation further indicates that the representative error significantly affects the simulated outcomes of random components of radar rainfall uncertainty.

The spatial and temporal correlation coefficients for group C scenarios are listed in Tables 8 and 9. Because there are only two radar pixels (pixels 15 and 25) in this scenario group, the spatial dependence refers to the correlation between them. The correlation coefficients were estimated by the Gaussian and t copulas simulations (see Table 8). There was no significant change in correlation coefficients among the eight scenarios for both the Gaussian and $\mathrm{t}$ copulas cases, indicating a limited influence of gauge representative error on simulating the spatial dependence of radar rainfall uncertainty. In terms of temporal correlation, we can see a slight growth of correlation coefficients from scenario $\mathrm{C} 1$ to scenario $\mathrm{C} 2$, but this trend is not continuous from $\mathrm{C} 2$ to $\mathrm{C} 8$. 
TABLE 6. Shape parameters of the GEV model for different radar pixels and scenarios.

\begin{tabular}{ccc}
\hline \hline Radar pixel & Scenario B1 & Scenario B2 \\
\hline 6 & 0.30 & 0.24 \\
9 & 0.40 & 0.23 \\
12 & 0.22 & 0.18 \\
15 & 0.50 & 0.35 \\
16 & 0.40 & 0.24 \\
19 & 0.30 & 0.24 \\
22 & 0.42 & 0.31 \\
25 & 0.33 & 0.27 \\
27 & 0.25 & 0.23 \\
\hline
\end{tabular}

\section{Impact of gauge representative error on rainfall uncertainty bands}

The uncertainty bands were derived by an MDEG model for all scenarios covering a period of 1 month. The MDEG was used to generate a large number (500 in this study) of rainfall ensemble members to represent the uncertainty associated with radar rainfall measurements. To quantify the variation of the uncertainty band, a dispersion indicator is defined to measure the variability of the generated ensemble members:

$$
\delta=\frac{1}{\mathrm{nt}} \sum_{i=1}^{\mathrm{nt}}\left[P_{95}(t)-P_{5}(t)\right] \text { for } P_{50}(t)>0,
$$

where $P_{95}(t), P_{5}(t)$, and $P_{50}(t)$ are the 95th, 5th, and 50th percentiles, respectively, of the ensemble members at time $t$. In addition, "nt" is the number of time steps corresponding to $P_{50}$ larger than zero. The dispersion indicators of the uncertainty bands using two copula models for all scenarios are listed in Tables 10 and 11 . To compare the indicators among different scenarios, only the simulated results of pixels 15 and 25 are shown as they are considered in all scenarios.

Theoretically speaking, the spread indicators of the group A scenarios should be close to each other because all of the configurations of the MDEG model used in these scenarios are exactly the same. The only differences among these scenarios are the locations of rain gauges within the two radar pixels. However, an obvious change of spread indicators among these scenarios was observed, as is shown in Table 10. Taking the Gaussian method and pixel 15 for example, the maximum and minimum spreads are 1.13 and 0.77 , respectively.

A comparison of uncertainty spreads between scenarios B1 and B2 is also listed in Table 10. The spread indicators are all considerably larger in scenario B1 in both pixels and with both copula methods. The $95 \%$ uncertainty bands of both scenarios, together with radar rainfall estimates, are delineated in Fig. 10. The ensemble members can be generated using Gaussian and t copula models. According to the above findings, the gauge representative error has limited influence on the spatial dependence of radar rainfall uncertainty, so the uncertainty band is illustrated for the Gaussian copula approach only. In general, Fig. 10 shows excellent agreement between both uncertainty bands for shape. The uncertainty bandwidth of scenario B1 is larger than that of scenario B2. In other words, the estimated radar rainfall uncertainty of the two-gauge-density scenario is smaller than that of the one-gauge-density scenario.

The spread indicators for group $\mathrm{C}$ scenarios are listed in Table 11. A general declining trend can be observed from scenarios $\mathrm{C} 1-\mathrm{C} 8$. The ratios between the indicators of scenarios $\mathrm{C} 2-\mathrm{C} 8$ and scenario $\mathrm{C} 1$ were calculated. There was no significant change in the spreads for
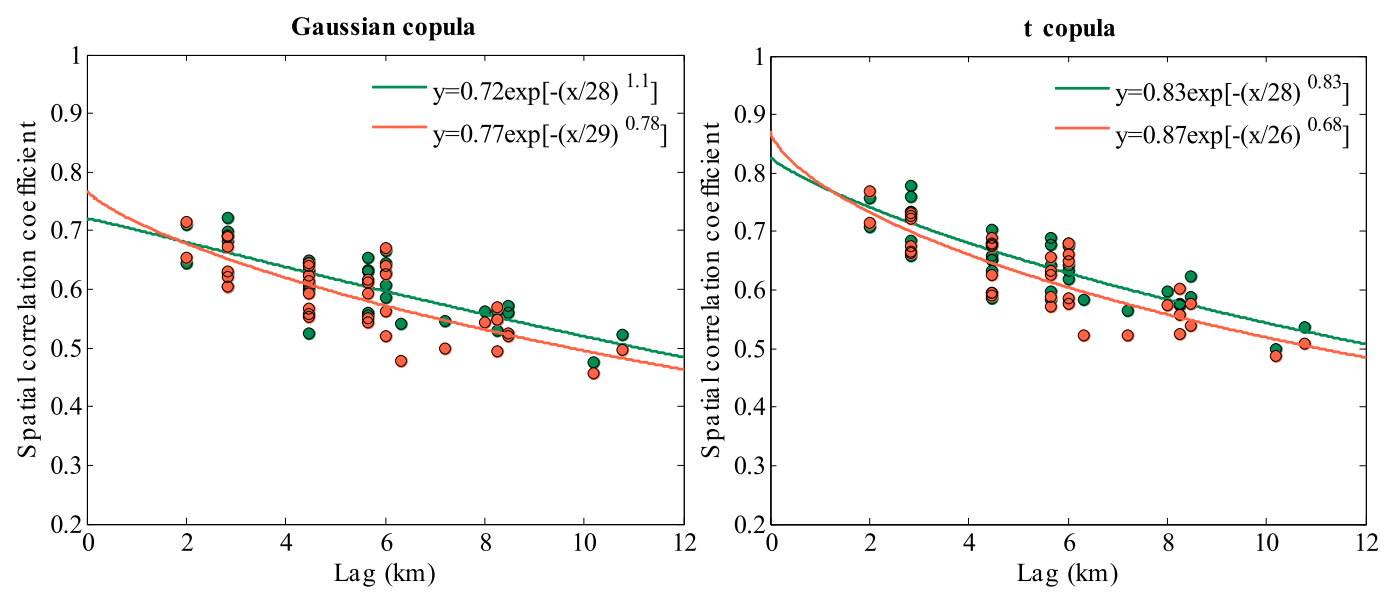

FIG. 7. The relationship between the spatial correlation coefficients of the radar rainfall errors and lag distances using (left) Gaussian and (right) t copulas. The scatterpoints represent correlation coefficients of scenarios B1 and B2; both scenarios are fitted with three-parameter exponential models (green line for B1, red line for B2). 

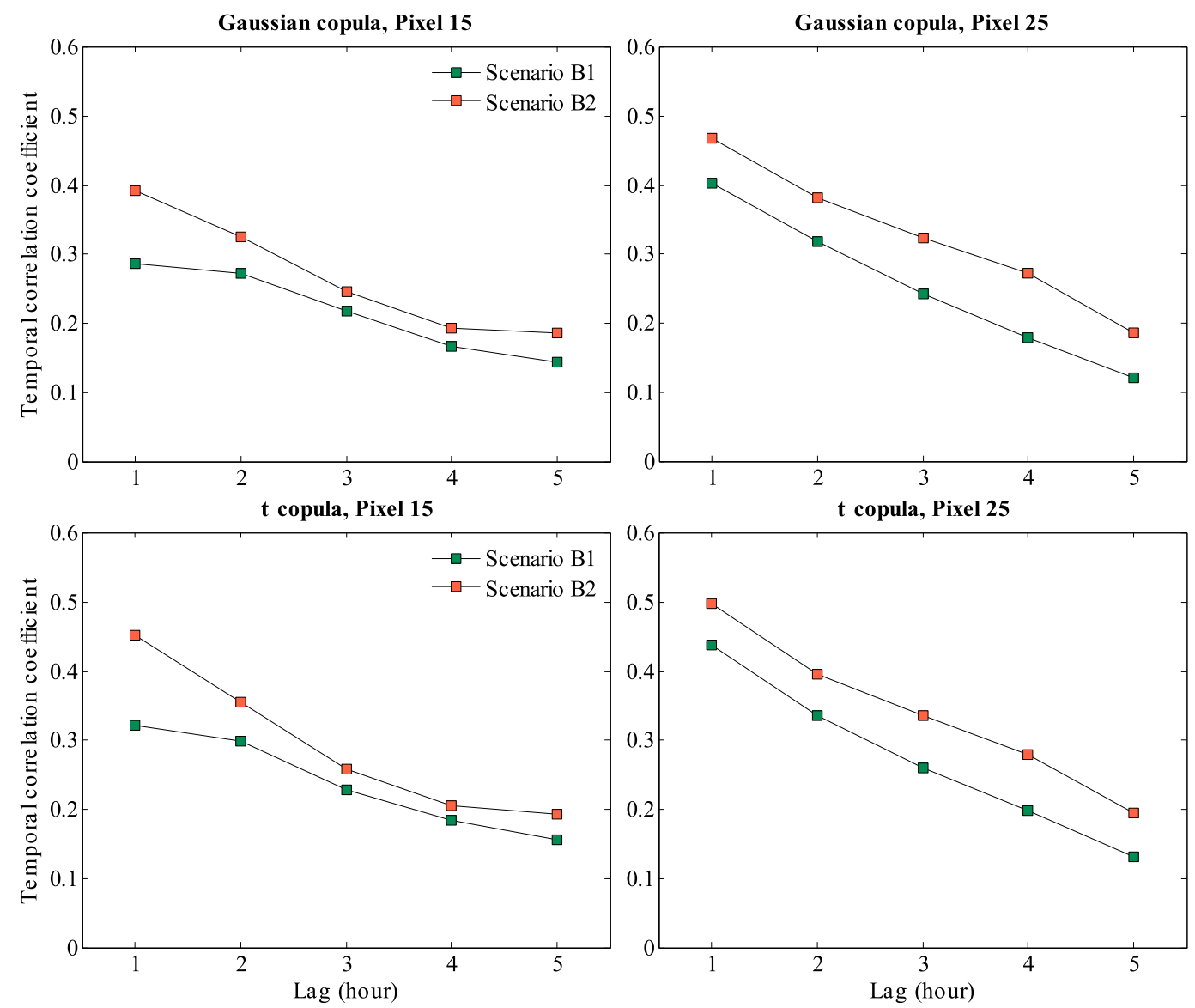

FIG. 8. The relationship between temporal correlation coefficients and lag hours for pixels (left) 15 and (right) 25 .

different copula methods in the same radar pixel. Therefore, the averaged values of both copula methods were used to calculate the ratio. There is a rapid drop of spread indicators from scenario $\mathrm{C} 1$ to $\mathrm{C} 2$, while only a slow decrease from $\mathrm{C} 2$ to $\mathrm{C} 8$ is evident. For instance, the uncertainty spread in scenario $\mathrm{C} 2$ is only $83 \%$ of scenario C1's in radar pixel 15 , while scenario C8's spread accounts for almost $78 \%$ of the uncertainty in scenario C1. As mentioned above, the differences in estimated radar rainfall uncertainties between scenario $\mathrm{C} 1$ and

TABLE 7. Rank correlation coefficients between radar and gauge rainfall measurements for different radar pixels and scenarios.

\begin{tabular}{ccc}
\hline Scenario & Pixel 15 & Pixel 25 \\
\hline C1 & 0.56 & 0.59 \\
C2 & 0.60 & 0.58 \\
C3 & 0.62 & 0.60 \\
C4 & 0.63 & 0.60 \\
C5 & 0.64 & 0.60 \\
C6 & 0.65 & 0.61 \\
C7 & 0.66 & 0.61 \\
C8 & 0.66 & 0.62 \\
\hline
\end{tabular}

scenario $\mathrm{C} 8$ can be attributed to the gauge representative uncertainty. Based on the above findings, it is worth remarking that the redundancy analysis of weather radar and rain gauge rainfall measurements is a promising method for rain gauge network design. In Fig. 11, the percentages of the gauge representative uncertainty (GRP) toward radar rainfall uncertainty are shown for different locations and copulas. The uncertainty percentage is estimated using the following equation:

$$
\mathrm{GRP}=\left(\delta_{\mathrm{C} 1}-\delta_{\mathrm{C} 8}\right) \times 100 / \delta_{\mathrm{C} 8} .
$$

It is observed that as much as $30 \%$ rainfall uncertainty actually stems from the gauge representative error. In addition, the fact that using different copulas produces slightly different percentages reveals the radar rainfall uncertainty model also has an impact on this conclusion.

\section{Discussion}

Through comparing three group scenarios regarding their behaviors in marginal distribution, spatial dependence, temporal dependence, and uncertainty bands 

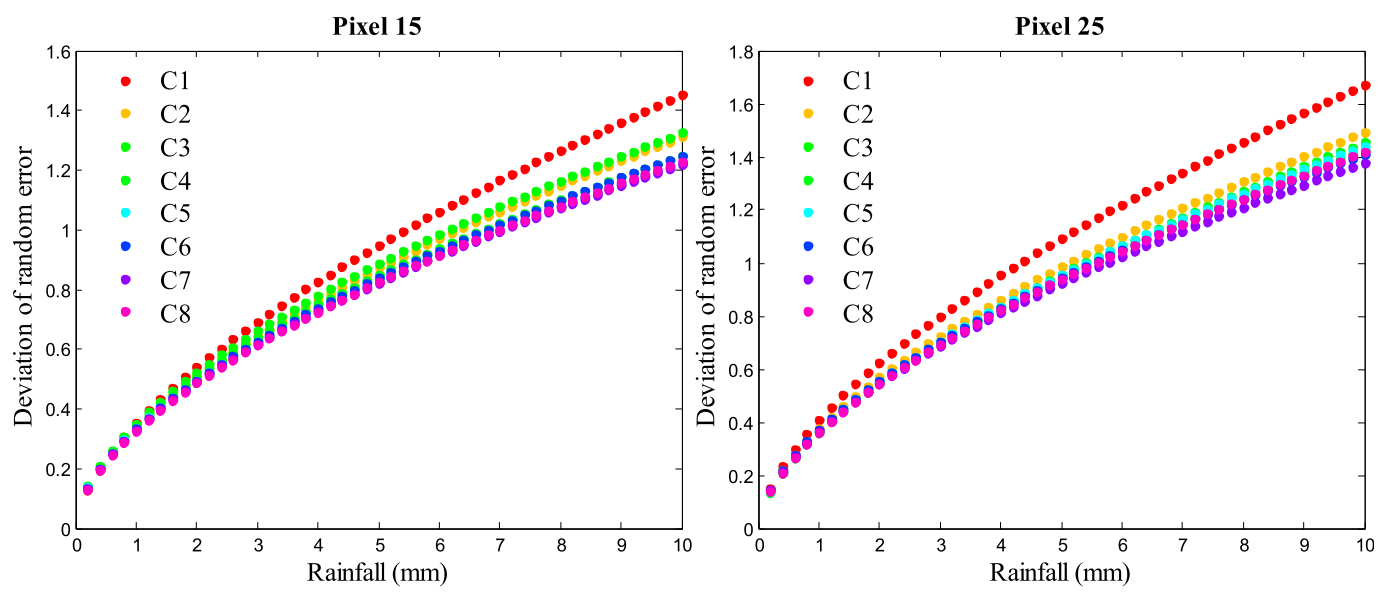

FIG. 9. Deviation of random error of hourly radar rainfall estimates for radar pixels (left) 15 and (right) 25.

of radar rainfall uncertainty, we found many significant results. However, we must consider whether these findings can answer the major questions raised by this study. Specifically, does the gauge representative error have a substantial effect on radar rainfall uncertainty analysis? If yes, what is the percentage of the derived radar rainfall uncertainty that could be induced by the gauge representative error rather than by the real radar rainfall uncertainty? Finally, what can we do to remove or correct for this effect when modeling the radar rainfall uncertainty based on gauge measurements?

In most current radar rainfall uncertainty analysis methods, the rain gauge located within the radar pixel is paired and correlated with the corresponding radar pixel, and the point gauge measurement is considered to be the "true" areal rainfall for the given radar pixel. According to the simulated results in group A, we find the uncertainty spreads significantly with a small shift in gauge location within a radar pixel (with an area of $2 \times$ $2 \mathrm{~km}^{2}$ ). Moreover, from the group C scenarios, we conclude that the correlation between radar and gauge rainfall amounts increases considerably with the number of gauges within the radar pixel, indicating the

TABLE 8. Spatial correlation coefficients of radar rainfall errors between radar pixels 15 and 25 .

\begin{tabular}{ccc}
\hline \hline Scenario & Gaussian copula & t copula \\
\hline C1 & 0.61 & 0.64 \\
C2 & 0.60 & 0.62 \\
C3 & 0.64 & 0.68 \\
C4 & 0.64 & 0.67 \\
C5 & 0.61 & 0.64 \\
C6 & 0.61 & 0.65 \\
C7 & 0.63 & 0.66 \\
C8 & 0.63 & 0.67 \\
\hline
\end{tabular}

discrepancy between radar and gauge datasets is not induced only by radar rainfall measurements. In other words, the gauge representative error indeed affects the discrepancy between radar and gauge rainfall amounts and an increase in gauge density is an effective way of reducing the gauge representative error.

There may be concern about the rain gauge merging method that calculates integrated rainfall. In the group $\mathrm{B}$ and $\mathrm{C}$ scenarios, we used a simple averaging scheme if more than one gauge existed in a radar pixel. More complicated methods such as interpolation schemes may be better. Considering the limited number of gauges (from two to eight), many complicated interpolation algorithms (e.g., kriging, spline, and trend) cannot be well implemented. To avoid the possible contamination from an averaging process, this study used a long-term historic dataset and all analysis courses were carried out in multiple locations using multiple gauge combinations. Because the conclusions drawn from the group B and C scenarios are quite consistent, we believe that a scheme that reflects gauge representative errors in various degrees of the averaging process can be acceptable. To sum up, the gauge representative error indeed induces individual discrepancies between radar and gauge rainfall measurements in the Brue catchment. Due to the poor gauge density in more realistic catchments, a more serious point-to-area error could have a greater impact on radar rainfall uncertainty analysis.

In modeling the radar rainfall uncertainty attributed to MDEG, one can see that the gauge representative error has a significant effect on the marginal distribution and slightly affects the temporal dependence of radar rainfall uncertainty. With these effects, the spread of rainfall uncertainty bands remarkably drops with the increase in gauge density. In other words, a considerable percentage of the estimated uncertainty comes from the 
TABLE 9. Temporal correlation coefficients for different lag hours (hours 1-5) and radar pixels.

\begin{tabular}{|c|c|c|c|c|c|c|c|c|c|c|}
\hline \multirow[b]{2}{*}{ Scenario } & \multicolumn{5}{|c|}{ Pixel 15} & \multicolumn{5}{|c|}{ Pixel 25} \\
\hline & $1 \mathrm{~h}$ & $2 \mathrm{~h}$ & $3 \mathrm{~h}$ & $4 \mathrm{~h}$ & $5 \mathrm{~h}$ & $1 \mathrm{~h}$ & $2 \mathrm{~h}$ & $3 \mathrm{~h}$ & $4 \mathrm{~h}$ & $5 \mathrm{~h}$ \\
\hline $\mathrm{C} 1$ & 0.42 & 0.34 & 0.27 & 0.17 & 0.17 & 0.39 & 0.28 & 0.22 & 0.19 & 0.14 \\
\hline $\mathrm{C} 2$ & 0.44 & 0.35 & 0.27 & 0.18 & 0.19 & 0.42 & 0.30 & 0.24 & 0.20 & 0.15 \\
\hline $\mathrm{C} 3$ & 0.41 & 0.33 & 0.25 & 0.17 & 0.20 & 0.47 & 0.36 & 0.30 & 0.26 & 0.21 \\
\hline $\mathrm{C} 4$ & 0.43 & 0.33 & 0.27 & 0.18 & 0.20 & 0.45 & 0.34 & 0.30 & 0.24 & 0.20 \\
\hline $\mathrm{C} 5$ & 0.44 & 0.34 & 0.28 & 0.20 & 0.21 & 0.46 & 0.33 & 0.31 & 0.25 & 0.21 \\
\hline C6 & 0.44 & 0.34 & 0.27 & 0.20 & 0.21 & 0.45 & 0.32 & 0.30 & 0.25 & 0.20 \\
\hline $\mathrm{C} 7$ & 0.44 & 0.34 & 0.27 & 0.20 & 0.20 & 0.45 & 0.32 & 0.30 & 0.25 & 0.20 \\
\hline $\mathrm{C} 8$ & 0.43 & 0.32 & 0.26 & 0.19 & 0.19 & 0.44 & 0.32 & 0.30 & 0.25 & 0.19 \\
\hline
\end{tabular}

gauge representative error. Specifically, the spread indicators of the uncertainty bands of scenario C8 accounted for $79 \%$ and $89 \%$ of $\mathrm{C} 1$ for two different locations (see Table 11). The difference is mainly caused by the gauge representative uncertainty because only gauge density varies among these scenarios. Admittedly, the average rainfall of eight gauges in a radar pixel still cannot reflect the true areal rainfall in that pixel and, consequently, the gauge representative error inevitably exists. However, it can be seen that the difference in uncertainty spread is significant when gauge density grows from one to two and then decreases for each additional rain gauge. For instance, the spread indicators of scenario $\mathrm{C} 7$ and $\mathrm{C} 8$ are almost the same. Therefore, it is credible to regard scenario $\mathrm{C} 8$ as a reference scenario and its estimated uncertainty differences with scenario $\mathrm{C} 1$ can to some extent reflect the representative-induced pseudouncertainty.

It is a challenge to model radar rainfall uncertainty considering the effects of gauge representative error. Apparently, increases in gauge numbers or decreases in radar spatial scale are two direct ways to compensate for the radar-gauge inconsistent-scale conundrum. Once a large number of gauges becomes available, the geostatistical techniques such as rainfall interpolation are a possible solution to estimating the mean areal rainfall.

TABLE 10. Spread of uncertainty bands of group A and B scenarios.

\begin{tabular}{cccccc}
\hline \hline & \multicolumn{2}{c}{ Pixel 15 } & & \multicolumn{2}{c}{ Pixel 25 } \\
\cline { 2 - 3 } \cline { 5 - 6 } Scenario & Gaussian & t copula & & Gaussian & t copula \\
\hline A1 & 0.81 & 0.83 & & 1.16 & 1.16 \\
A2 & 1.13 & 1.13 & & 1.31 & 1.32 \\
A3 & 0.79 & 0.80 & & 1.22 & 1.22 \\
A4 & 0.77 & 0.78 & & 1.43 & 1.42 \\
A5 & 0.88 & 0.89 & & 1.24 & 1.24 \\
A6 & 0.85 & 0.84 & & 1.20 & 1.23 \\
A7 & 0.81 & 0.81 & & 1.14 & 1.17 \\
A8 & 0.83 & 0.84 & & 1.16 & 1.16 \\
B1 & 0.79 & 0.74 & & 1.02 & 1.03 \\
B2 & 0.56 & 0.57 & & 0.88 & 0.86 \\
\hline
\end{tabular}

Thus, the gauge representative error could be investigated by comparing a single gauge's rainfall amount and the mean areal rainfall. However, it is impractical to obtain high-density rain gauge network (say, one gauge per radar pixel) or high spatial resolution radar (say, $<1 \mathrm{~km}$ ) results in most real catchments. To establish an experimental station specially for studying gauge representative error in the given study area may be an alternative solution. As shown in this study, two superdense rain gauge networks were set up within two radar pixels. Eight rain gauges were evenly configured in the corresponding radar pixel. The results show that twogauge density seems to be enough for a $2-\mathrm{km}$-scale case to identify the gauge representative error. Through analyzing the characteristics of the relationships between gauge representative error and radar rainfall uncertainty in the experimental area, one may extend the conclusion to the whole area. However, there may be varying degrees of gauge representative errors in different locations (as shown in this study), which requires further investigation. If the spatial correlation of radar rainfall is known, the radar-gauge difference variance can be partitioned into the radar-rainfall estimation error variance and the representative error variance based on the aforementioned EVS scheme. The integration of such schemes with the proposed MDEG method is a promising way to fully solve the representative error issue, which will be explored in a future study.

\section{Conclusions}

In modeling the radar rainfall uncertainty, rain gauge measurement is generally regarded as an areal "true" rainfall. However, the inconsistent scales between radar and gauge may introduce a new uncertainty, which is erroneously identified as radar rainfall uncertainty itself. By leveraging the strengths of the proposed radar rainfall uncertainty model, 3 groups with 18 scenarios were designed to investigate the impacts of gauge representative error on the radar rainfall uncertainty model using 
TABLE 11. Spread of uncertainty bands of group C scenarios. The ratio refers to the mean spread indicators of scenarios $\mathrm{C} 2-\mathrm{C} 8$ to those of scenario $\mathrm{C} 1$.

\begin{tabular}{|c|c|c|c|c|c|c|}
\hline \multirow[b]{2}{*}{ Scenario } & \multicolumn{3}{|c|}{ Pixel 15} & \multicolumn{3}{|c|}{ Pixel 25} \\
\hline & Gaussian & t copula & Ratio (\%) & Gaussian & t copula & Ratio (\%) \\
\hline $\mathrm{C} 1$ & 0.71 & 0.70 & - & 1.01 & 0.99 & - \\
\hline $\mathrm{C} 2$ & 0.59 & 0.58 & 83 & 0.92 & 0.92 & 92 \\
\hline C3 & 0.58 & 0.57 & 82 & 0.94 & 0.94 & 94 \\
\hline $\mathrm{C} 4$ & 0.59 & 0.60 & 84 & 0.91 & 0.91 & 91 \\
\hline C5 & 0.58 & 0.57 & 82 & 0.92 & 0.91 & 92 \\
\hline C6 & 0.57 & 0.57 & 81 & 0.91 & 0.91 & 91 \\
\hline $\mathrm{C} 7$ & 0.56 & 0.56 & 79 & 0.91 & 0.89 & 90 \\
\hline C8 & 0.55 & 0.55 & 78 & 0.89 & 0.89 & 89 \\
\hline
\end{tabular}

long-term datasets (almost 7 years) from the Brue catchment in the United Kingdom. Despite the fact that the radar and rain gauges do not record up-to-date rainfall data, the Brue catchment is still an ideal area for this study. The rain gauge network was designed by considering the locations of radar pixels, thus ensuring the density and distribution of the rain gauges are quite suitable for analyzing the radar-gauge inconsistent-scale issue, and also the radar and gauge datasets are trustworthy because of careful quality control (Bringi et al. 2011; Moore et al. 2000). In summary, there are four main findings in this study. First, a considerable part of the uncertainty estimated by the gauge-based radar rainfall uncertainty model actually stems from the gauge representative error. The gauge representative error can induce as much as $21 \%$ differences in uncertainty

\section{Gaussian copula, Pixel 15}
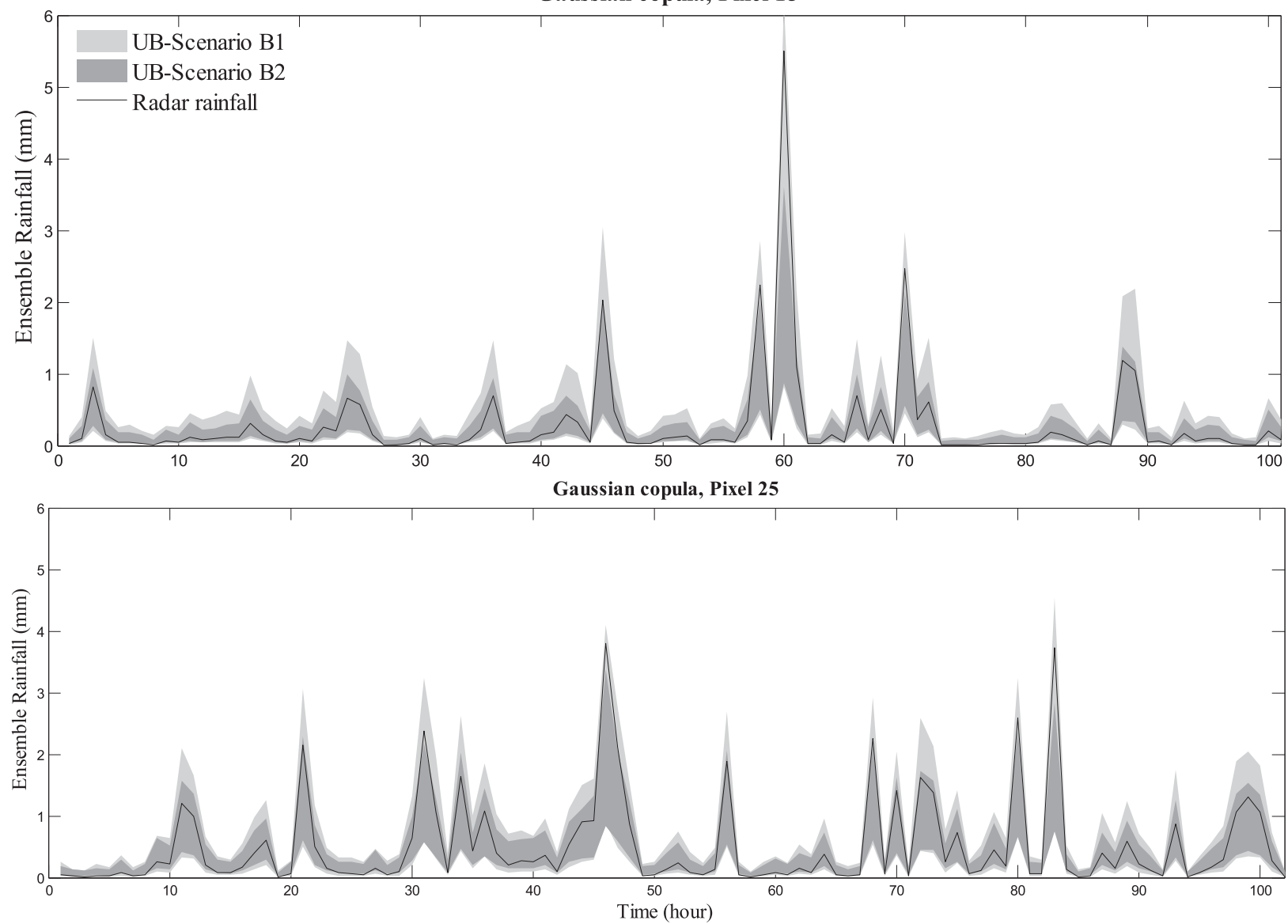

FIG. 10. Radar rainfall uncertainty bands for scenarios B1 and B2 at hourly temporal scale. 


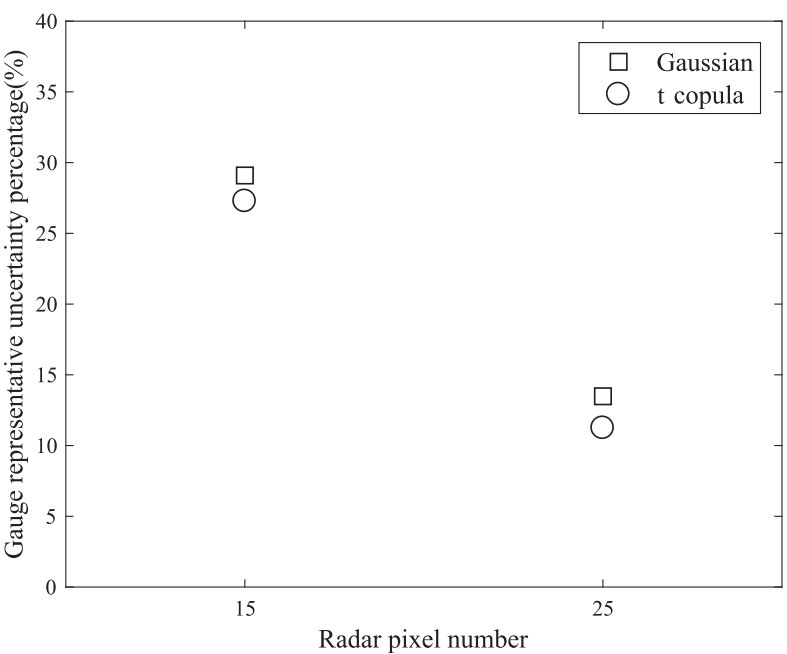

FIG. 11. Percentages of gauge representative uncertainty toward radar rainfall uncertainty for different locations and copulas.

spreads between two scenarios (refer to scenarios C1 and C8). Second, the marginal distribution of the radar rainfall uncertainty is best fitted by the GEV model in the Brue catchment, and this conclusion is not affected by gauge representative error. Third, the spatial dependence of the radar rainfall uncertainty is quite consistent for various gauge densities. Finally, at $2-\mathrm{km}$ radar scale, the uncertainty induced by gauge representative error will be significantly reduced by shifting from onegauge density to two-gauge density, and it drops only slowly with a higher gauge density.

Due to the complexity of gauge representative error, there are still some unsolved problems. The representative error under different scales may cause different degrees of contamination in the radar rainfall uncertainty modeling. Because a 2-km-scale radar pixel was used herein, it may be useful to further explore different radar spatial scales. Moreover, as the historical rainfall records that are larger than $10 \mathrm{~mm} \mathrm{~h}^{-1}$ are not sufficient to support a reliable fit for the relationship between rainfall rates and their uncertainties, the corresponding relationship is established only using the rainfall rates that are smaller than $10 \mathrm{~mm} \mathrm{~h}^{-1}$, but it is extended to the heavy rainfall rates. The possible influence of this compromise on the proposed scheme will be investigated in future work. In addition, some external factors such as topographic fluctuations could affect the gauge representative error. Because the terrain of the Brue catchment has small variations ( $250 \mathrm{~m}$ or less), this issue is not thought to be significant in this study. However, for hilly areas, solely increasing the gauge density may not absolutely remit the gauge representative effect. Because of the poor gauge density in realistic catchments, the resulting more serious point-to-area error could have additional awkward consequences for radar rainfall uncertainty analyses. For example, a rain gauge network (with 50 gauges) in Yorkshire was used to analyze the radar rainfall uncertainty (Dai et al. 2015). The gauge density was only about 1 gauge per $200 \mathrm{~km}^{2}$. For this reason, it is hoped that additional catchments with diverse climate and geographical conditions and additional radars operating at various scales will be explored by the research community to further verify and improve the proposed scheme. Moreover, because radar rainfall uncertainty is an important source of hydrological model uncertainty, it is of interest to know how the representative error will eventually affect the uncertainty analysis of hydrological models, which will be explored in a future study.

Acknowledgments. This study is supported by the National Natural Science Foundation of China (41771424 and 41501429), the Newton Fund via Natural Environment Research Council (NERC), the Economic and Social Research Council (ESRC) (NE/N012143/1), and the University Natural Science Project of Jiangsu Province (16KJA170001).

\section{REFERENCES}

AghaKouchak, A., A. Bárdossy, and E. Habib, 2010: Conditional simulation of remotely sensed rainfall data using a nonGaussian v-transformed copula. Adv. Water Resour., 33, 624-634, https://doi.org/10.1016/j.advwatres.2010.02.010.

Anagnostou, E. N., W. F. Krajewski, and J. Smith, 1999: Uncertainty quantification of mean-areal radar-rainfall estimates. J. Atmos. Oceanic Technol., 16, 206-215, https:// doi.org/10.1175/1520-0426(1999)016<0206:UQOMAR > 2.0.CO;2.

Barnston, A. G., 1991: An empirical method of estimating raingage and radar rainfall measurement bias and resolution. J. Appl. Meteor., 30, 282-296, https://doi.org/10.1175/1520-0450(1991) $030<0282$ :AEMOER $>2.0 . \mathrm{CO} ; 2$.

Bell, V., and R. Moore, 2000: The sensitivity of catchment runoff models to rainfall data at different spatial scales. Hydrol. Earth Syst. Sci., 4, 653-667, https://doi.org/10.5194/hess-4-653-2000.

Borga, M., F. Tonelli, R. J. Moore, and H. Andrieu, 2002: Longterm assessment of bias adjustment in radar rainfall estimation. Water Resour. Res., 38, 1226, https://doi.org/10.1029/ 2001 WR000555.

Bringi, V., M. Rico-Ramirez, and M. Thurai, 2011: Rainfall estimation with an operational polarimetric C-band radar in the United Kingdom: Comparison with a gauge network and error analysis. J. Hydrometeor., 12, 935-954, https://doi.org/10.1175/ JHM-D-10-05013.1.

Ciach, G. J., 2003: Local random errors in tipping-bucket rain gauge measurements. J. Atmos. Oceanic Technol., 20, 752759, https://doi.org/10.1175/1520-0426(2003)20<752:LREITB > 2.0.CO;2.

-, and W. F. Krajewski, 1999a: On the estimation of radar rainfall error variance. Adv. Water Resour., 22, 585-595, https://doi.org/10.1016/S0309-1708(98)00043-8. 
_ and _ 1999b: Radar-rain gauge comparisons under observational uncertainties. J. Appl. Meteor., 38, 1519-1525, https://doi.org/10.1175/1520-0450(1999)038<1519:RRGCUO> 2.0.CO;2.

- - , and G. Villarini, 2007: Product-error-driven uncertainty model for probabilistic quantitative precipitation estimation with NEXRAD data. J. Hydrometeor., 8, 13251347, https://doi.org/10.1175/2007JHM814.1.

Collier, C., and P. James, 1986: On the development of an integrated weather radar data processing system. Preprints, $23 \mathrm{rd}$ Conf. on Radar Meteorology, Snowmass, CO, Amer. Meteor. Soc., 95-98.

Dai, Q., and D. Han, 2014: Exploration of discrepancy between radar and gauge rainfall estimates driven by wind fields. Water Resour. Res., 50, 8571-8588, https://doi.org/10.1002/ 2014WR015794.

, - — M. A. Rico-Ramirez, and T. Islam, 2013: The impact of raindrop drift in a three-dimensional wind field on a radargauge rainfall comparison. Int. J. Remote Sens., 34, 7739-7760, https://doi.org/10.1080/01431161.2013.826838.

$-, \ldots, \ldots$, and $\_, 2014 \mathrm{a}$ : Modelling radar-rainfall estimation uncertainties using elliptical and Archimedean copulas with different marginal distributions. Hydrol. Sci. J., 59, 19922008, https://doi.org/10.1080/02626667.2013.865841.

$-\ldots,-, \ldots$, and P. K. Srivastava, 2014b: Multivariate distributed ensemble generator: A new scheme for ensemble radar precipitation estimation over temperate maritime climate. J. Hydrol., 511, 17-27, https://doi.org/10.1016/ j.jhydrol.2014.01.016.

—, M. A. Rico-Ramirez, D. Han, T. Islam, and S. Liguori, 2015: Probabilistic radar rainfall nowcasts using empirical and theoretical uncertainty models. Hydrol. Processes, 29, 66-79, https://doi.org/10.1002/hyp.10133.

—, M. Bray, L. Zhuo, T. Islam, and D. Han, 2017: A scheme for rain gauge network design based on remotely sensed rainfall measurements. J. Hydrometeor., 18, 363-379, https://doi.org/ 10.1175/JHM-D-16-0136.1.

Germann, U., M. Berenguer, D. Sempere-Torres, and M. Zappa, 2009: REAL-Ensemble radar precipitation estimation for hydrology in a mountainous region. Quart. J. Roy. Meteor. Soc., 135, 445-456, https://doi.org/10.1002/ qj.375.

Habib, E., and L. Qin, 2013: Application of a radar-rainfall uncertainty model to the NWS multi-sensor precipitation estimator products. Meteor. Appl., 20, 276-286, https://doi.org/ 10.1002/met.301.

— W W. F. Krajewski, V. Nespor, and A. Kruger, 1999: Numerical simulation studies of rain gage data correction due to wind effect. J. Geophys. Res., 104, 19723-19733, https://doi.org/ 10.1029/1999JD900228.

_- G. G. Ciach, and W. F. Krajewski, 2004: A method for filtering out raingauge representativeness errors from the verification distributions of radar and raingauge rainfall. Adv. Water Resour., 27, 967-980, https://doi.org/10.1016/ j.advwatres.2004.08.003.

— , A. V. Aduvala, and E. A. Meselhe, 2008: Analysis of radarrainfall error characteristics and implications for streamflow simulation uncertainty. Hydrol. Sci. J., 53, 568-587, https:// doi.org/10.1623/hysj.53.3.568.

Hall, W., M. A. Rico-Ramirez, and S. Krämer, 2015: Classification and correction of the bright band using an operational C-band polarimetric radar. J. Hydrol., 531, 248-258, https://doi.org/ 10.1016/j.jhydrol.2015.06.011.
Hasan, M. M., A. Sharma, F. Johnson, G. Mariethoz, and A. Seed, 2014: Correcting bias in radar $Z-R$ relationships due to uncertainty in point rain gauge networks. J. Hydrol., 519, 16681676, https://doi.org/10.1016/j.jhydrol.2014.09.060.

$\ldots,-,-, \ldots$, and,$- 2016 \mathrm{a}$ : Merging radar and in situ rainfall measurements: An assessment of different combination algorithms. Water Resour. Res., 52, 8384-8398, https:// doi.org/10.1002/2015WR018441.

$\_,-$G. Mariethoz, F. Johnson, and A. Seed, 2016b: Improving radar rainfall estimation by merging point rainfall measurements within a model combination framework. Adv. Water Resour., 97, 205-218, https://doi.org/10.1016/ j.advwatres.2016.09.011.

Kirstetter, P.-E., G. Delrieu, B. Boudevillain, and C. Obled, 2010: Toward an error model for radar quantitative precipitation estimation in the Cévennes-Vivarais region, France. J. Hydrol., 394, 28-41, https://doi.org/10.1016/ j.jhydrol.2010.01.009.

—_, J. J. Gourley, Y. Hong, J. Zhang, S. Moazamigoodarzi, C. Langston, and A. Arthur, 2015: Probabilistic precipitation rate estimates with ground-based radar networks. Water Resour. Res., 51, 1422-1442, https://doi.org/ 10.1002/2014WR015672.

Krajewski, W. F., G. Villarini, and J. A. Smith, 2010: Radar-rainfall uncertainties: Where are we after thirty years of effort? Bull. Amer. Meteor. Soc., 91, 87-94, https://doi.org/10.1175/ 2009BAMS2747.1.

Marshall, J., W. Hitschfeld, and K. Gunn, 1955: Advances in radar weather. Advances in Geophysics, Vol. 2, Academic Press, 1-56, https://doi.org/10.1016/S0065-2687(08)60310-6.

Michelson, D. B., 2004: Systematic correction of precipitation gauge observations using analyzed meteorological variables. J. Hydrol., 290, 161-177, https://doi.org/10.1016/ j.jhydrol.2003.10.005.

Moore, R., D. Jones, D. Cox, and V. Isham, 2000: Design of the HYREX raingauge network. Hydrol. Earth Syst. Sci., 4, 521530, https://doi.org/10.5194/hess-4-521-2000.

Nikahd, A., M. Hashim, and M. J. Nazemosadat, 2016: A review of uncertainty sources on weather ground-based radar for rainfall estimation. Appl. Mech. Mater., 254-271, https://doi.org/ 10.4028/www.scientific.net/AMM.818.254.

Qi, Y., J. Zhang, P. Zhang, and Q. Cao, 2013: VPR correction of bright band effects in radar QPEs using polarimetric radar observations. J. Geophys. Res. Atmos., 118, 3627-3633, https:// doi.org/10.1002/jgrd.50364.

Rico-Ramirez, M., I. Cluckie, G. Shepherd, and A. Pallot, 2007: A high-resolution radar experiment on the island of Jersey. Meteor. Appl., 14, 117-129, https://doi.org/10.1002/ met.13.

—, S. Liguori, and A. Schellart, 2015: Quantifying radar-rainfall uncertainties in urban drainage flow modelling. J. Hydrol., 528, 17-28, https://doi.org/10.1016/j.jhydrol.2015.05.057.

Sandford, C., 2015: Correcting for wind drift in high resolution radar rainfall products: A feasibility study. J. Hydrol., 531, 284-295, https://doi.org/10.1016/j.jhydrol.2015.03.023.

Seo, B. C., L. K. Cunha, and W. F. Krajewski, 2013: Uncertainty in radar-rainfall composite and its impact on hydrologic prediction for the eastern Iowa flood of 2008. Water Resour. Res., 49, 2747-2764, https://doi.org/10.1002/wrcr.20244.

Villarini, G., P. V. Mandapaka, W. F. Krajewski, and R. J. Moore, 2008: Rainfall and sampling uncertainties: A rain gauge perspective. J. Geophys. Res., 113, D11102, https://doi.org/ 10.1029/2007JD009214. 
_- B.-C. Seo, F. Serinaldi, and W. F. Krajewski, 2014: Spatial and temporal modeling of radar rainfall uncertainties. Atmos. Res., 135-136, 91-101, https://doi.org/10.1016/ j.atmosres.2013.09.007.

Wang, Y., J. Zhang, P.-L. Chang, and Q. Cao, 2015: Radar vertical profile of reflectivity correction with TRMM observations using a neural network approach. J. Hydrometeor., 16, 22302247, https://doi.org/10.1175/JHM-D-14-0136.1.

Wheater, H., and Coauthors, 2000: Spatial-temporal rainfall fields: Modelling and statistical aspects. Hydrol. Earth Syst. Sci., 4, 581-601, https://doi.org/10.5194/hess-4-581-2000.
Wood, S., D. Jones, and R. Moore, 2000a: Accuracy of rainfall measurement for scales of hydrological interest. Hydrol. Earth Syst. Sci., 4, 531-543, https://doi.org/10.5194/hess-4-531-2000. , and - 2000b: Static and dynamic calibration of radar data for hydrological use. Hydrol. Earth Syst. Sci., 4, 545-554, https://doi.org/10.5194/hess-4-545-2000.

Xie, X., R. Evaristo, S. Troemel, P. Saavedra, C. Simmer, and A. Ryzhkov, 2016: Radar observation of evaporation and implications for quantitative precipitation and cooling rate estimation. J. Atmos. Oceanic Technol., 33, 1779-1792, https:// doi.org/10.1175/JTECH-D-15-0244.1. 